\title{
Ausencia de circulación de poliovirus en departamentos colombianos con coberturas vacunales inferiores a $80 \%$
}

\author{
María Mercedes González, ${ }^{1}$ Luis Sarmiento, ${ }^{2}$ Gloria Janneth Rey-Benito, ${ }^{3}$ \\ Leonardo Padilla, ${ }^{1}$ Alejandra María Giraldo ${ }^{1}$ y Jhon Carlos Castaño ${ }^{1}$
}

Forma de citar González MM, Sarmiento L, Rey-Benito GJ, Padilla L, Giraldo AM, Castaño JC. Ausencia de circulación de poliovirus en departamentos colombianos con coberturas vacunales inferiores a $80 \%$. Rev Panam Salud Publica. 2012;32(2):156-60.

RESUMEN El presente estudio se propuso explorar la posible circulación silente de poliovirus salvajes y derivados de la vacuna (VDPV, por sus siglas en inglés), en departamentos de Colombia con cobertura de vacunación para polio (OPV, por sus siglas en inglés) menor de $80 \%$. Se colectaron 52 muestras de aguas residuales que se concentraron mediante precipitación con polietilenglicol y cloruro de sodio. La detección viral se realizó mediante aislamiento y la identificación por neutralización del efecto citopático, así como mediante reacción en cadena de la polimerasa convencional y en tiempo real, posterior a la transcripción reversa (TR-RCP y rTR-RCP). Los poliovirus aislados se caracterizaron por secuenciación del gen VP1. En dos de las 52 muestras hubo presencia de poliovirus Sabin 2 con más de 99\% de similitud de secuencia con la cepa OPV Sabin 2. Se detectó circulación de enterovirus no polio en 17,3\% de las muestras. Los serotipos identificados correspondieron a coxsackievirus B1, echovirus 30 y echovirus 11. No se detectaron evidencias de circulación de VDPV ni poliovirus salvaje en los departamentos de Colombia con coberturas de OPV inferiores a 80\%.

Palabras clave Poliomielitis; vacuna antipolio oral; vacunas contra poliovirus; cobertura de vacunación; Colombia.

La poliomielitis es una enfermedad infecciosa causada por los tres serotipos del virus de la polio. No tiene tratamiento específico, pero se incluye en las enfermedades prevenibles por vacunación (1). En Colombia el último caso de infección por poliovirus salvaje se produjo en Arjona en 1991, después de que en el país se alcanzara buena inmunidad poblacional debida a la introducción de la vacuna

\footnotetext{
Universidad del Quindío, Armenia, Colombia. La correspondencia se debe dirigir a María Mercedes González. Correo electrónico: mmgonzalez@uniquindio.edu.co

2 Instituto de Medicina Tropical Pedro Kourí, La Habana, Cuba.

3 Instituto Nacional de Salud, Bogotá, Colombia.
}

antipoliomielítica oral de virus vivo atenuado (OPV, por sus siglas en inglés) en el programa regular de inmunización desde 1974 y al desarrollo de varias jornadas nacionales de vacunación. El esquema vacunal contra la poliomielitis consiste en tres dosis en el primer año de vida (a los 2, 4 y 6 meses) y dos refuerzos a los 18 meses y 5 años de edad, está disponible los cinco días hábiles de la semana en las instituciones de salud e incluye campañas de vacunación (2).

En Colombia entre los años 2004 y 2007 (período de referencia para este estudio), el programa de vacunación antipolio no logró coberturas útiles, definidas como iguales o superiores a $90 \%$ (3). El conflicto armado presente en las áreas seleccionadas para el estudio, a excepción de San Andrés y Providencia, constituyó un factor que limitó de manera considerable el acceso de la población a la vacunación, al igual que el difícil acceso a algunas zonas geográficas (4).

Estudios previos realizados en el departamento del Quindío, donde las coberturas de vacunación antipolio son superiores a $80 \%$, no detectaron circulación de poliovirus salvajes ni cepas "modificadas" originadas de las vacunales conocidas como "poliovirus derivados de la vacuna" (VDPV, por sus siglas en 
inglés). Contrariamente, en poblaciones con bajas coberturas suele persistir la circulación del poliovirus salvaje, fenómeno demostrado con la aparición de brotes de poliomielitis asociados a importación de poliovirus salvajes en Canadá en 1978, Holanda en los años 1978 y 1993, Estados Unidos en 1979, Bulgaria en 2001 y más recientemente en Tayikistán y Turkmenistán $(5,6)$. Los factores de riesgo para la circulación de VDPV parecen ser similares a los del virus salvaje, como se observa en algunos brotes causados por el poliovirus derivado de vacuna en países como Camboya, China, Haití, Filipinas, Indonesia, Madagascar, Myanmar, Níger, Nigeria y República Dominicana (7).

En este contexto, se realizó un estudio exploratorio de corte transversal, me- diante una encuesta de prevalencia de poliovirus en aguas residuales en nueve departamentos de Colombia con coberturas de vacuna de polio oral inferiores a 80\% durante el período 2004-2007 (cuadro 1). El objetivo fue determinar la posible circulación silente de poliovirus salvajes y derivados de la vacuna en departamentos de Colombia con cobertura de inmunización para polio menor de $80 \%$.

Por cada departamento se seleccionaron seis puntos de colección: tres en los sitios finales de descarga del alcantarillado de la capital y tres en los sitios finales de descarga de un municipio con baja cobertura vacunal en el departamento seleccionado $(n=54)$. Los sitios elegidos para el muestreo fueron aquellos donde convergieron la mayor cantidad de vertederos del municipio, teniendo como fuente de información a la entidad pública o privada encargada del manejo de los alcantarillados en el departamento respectivo. De cada punto de colección se tomó un litro de agua. Se excluyeron de la muestra dos vertederos contaminados con solventes orgánicos, por lo que se colectaron en total 52 litros de aguas residuales.

Las muestras fueron obtenidas directamente de los puntos de recolección según las recomendaciones de la OMS (8). La recuperación y concentración de virus se realizó mediante precipitación con polietilenglicol y cloruro de sodio, conforme a los principios generales del método descrito por Sobsey en 1987 (9).

Se inocularon por duplicado muestras de $200 \mu L$ de aguas residuales concentradas en dos líneas celulares: las células de rabdomiosarcoma humano (RD) y las

CUADRO 1. Cobertura de vacuna antipoliomielítica oral de virus vivo atenuado (OPV, por sus siglas en inglés), por departamentos y municipios, Colombia, 2004-2007

\begin{tabular}{|c|c|c|c|c|c|c|c|c|c|}
\hline $\begin{array}{c}\text { Año / Cobertura } \\
\text { nacional (\%) }\end{array}$ & Departamento & $\begin{array}{l}\text { Población } \\
<1 \text { año }\end{array}$ & $\begin{array}{c}\text { Cobertura } \\
(\%)\end{array}$ & Municipio capital & $\begin{array}{l}\text { Población } \\
<1 \text { año }\end{array}$ & $\begin{array}{c}\text { Cobertura } \\
(\%)\end{array}$ & Otro municipio & $\begin{array}{l}\text { Población } \\
<1 \text { año }\end{array}$ & $\begin{array}{c}\text { Cobertura } \\
(\%)\end{array}$ \\
\hline \multirow[t]{9}{*}{$2004(89)$} & Boyacá & 28968 & 79,56 & Tunja & 2816 & 105,3 & Ráquira & 219 & 51,1 \\
\hline & Caldas & 20578 & 72,84 & Manizales & 6353 & 86,6 & Neira & 545 & 83,9 \\
\hline & Cauca & 29871 & 70,82 & Popayán & 4700 & 92,8 & Piendamó & 660 & 101,4 \\
\hline & Guainía & 1171 & 39,62 & Puerto Inírida & 744 & 41,1 & ND & & \\
\hline & Guaviare & 3301 & 68,95 & San José & 1734 & 83,3 & El Retorno & 556 & 64,0 \\
\hline & Nariño & 36887 & 69,75 & Pasto & 8105 & 78,5 & Buesaco & 422 & 99,8 \\
\hline & Vichada & 2747 & 51,00 & Puerto Carreño & 531 & 74,2 & La Primavera & 421 & 32,8 \\
\hline & Risaralda & 18071 & 78,77 & Pereira & 8838 & 72,0 & Belén de Umbría & 555 & 99,8 \\
\hline & San Andrés y Providencia & 1499 & 66,78 & San Andrés & 1360 & 69,9 & Providencia & 139 & 36,7 \\
\hline \multirow[t]{9}{*}{$2005(93)$} & Boyacá & 29258 & 76,60 & Tunja & 2864 & 105,8 & Ráquira & 318 & 34,3 \\
\hline & Caldas & 2856 & 73,40 & Manizales & 5424 & 101,7 & Neira & 622 & 68,6 \\
\hline & Cauca & 30458 & 73,40 & Popayán & 3862 & 94,9 & Piendamó & 725 & 89,8 \\
\hline & Guainía & 1210 & 48,60 & Puerto Inírida & 400 & 97,3 & ND & & \\
\hline & Guaviare & 3391 & 65,90 & San José & 1531 & 96,2 & El Retorno & 594 & 65,0 \\
\hline & Nariño & 37591 & 65,90 & Pasto & 6362 & 119,4 & Buesaco & 427 & 83,8 \\
\hline & Vichada & 2819 & 46,50 & Puerto Carreño & 325 & 122,5 & La Primavera & 307 & 36,8 \\
\hline & Risaralda & 18401 & 69,80 & Pereira & 6966 & 86,4 & Belén de Umbría & 546 & 81,1 \\
\hline & San Andrés y Providencia & 1538 & 59,80 & San Andrés & 1206 & 70,6 & ND & & \\
\hline \multirow[t]{9}{*}{$2006(93,8)$} & Boyacá & 29902 & 72,60 & Tunja & 2864 & 105,8 & Ráquira & 318 & 34,3 \\
\hline & Caldas & 21237 & 69,10 & Manizales & 5424 & 101,7 & Neira & 622 & 68,6 \\
\hline & Cauca & 30823 & 77,40 & Popayán & 3862 & 94,9 & Piendamó & 725 & 89,8 \\
\hline & Guainía & 1208 & 63,00 & Puerto Inírida & 458 & 125,5 & ND & & \\
\hline & Guaviare & 3406 & 63,40 & San José & 1547 & 90,1 & El Retorno & 581 & 56,8 \\
\hline & Nariño & 3807 & 68,30 & Pasto & 6541 & 82,6 & Buesaco & 440 & 58,6 \\
\hline & Vichada & 2836 & 28,20 & Puerto Carreño & 350 & 120,9 & La Primavera & 318 & 29,6 \\
\hline & Risaralda & 18648 & 67,40 & Pereira & 7071 & 83,2 & Belén de Umbría & 545 & 81,1 \\
\hline & San Andrés y Providencia & 1292 & 69,10 & San Andrés & 1204 & 69,1 & ND & & \\
\hline \multirow[t]{9}{*}{$2007(92,8)$} & Boyacá & 29902 & 57,10 & Tunja & 2997 & 101,6 & Ráquira & 311 & 32,8 \\
\hline & Caldas & 21237 & 49,40 & Manizales & 5497 & 85,1 & Neira & 599 & 58,9 \\
\hline & Cauca & 30823 & 46,00 & Popayán & 3903 & 83,3 & Piendamó & 725 & 81,8 \\
\hline & Guainía & 1208 & 34,20 & Puerto Inírida & 457 & 89,9 & ND & & \\
\hline & Guaviare & 3406 & 51,40 & San José & 1555 & 103,2 & El Retorno & 583 & 56,8 \\
\hline & Nariño & 38071 & 46,90 & Pasto & 6463 & 91,7 & Buesaco & 438 & 68,9 \\
\hline & Vichada & 2836 & 23,10 & Puerto Carreño & 355 & 116,1 & La Primavera & 329 & 45,0 \\
\hline & Risaralda & 18648 & 49,20 & Pereira & 7058 & 76,4 & Belén de Umbría & 543 & 79,6 \\
\hline & San Andrés y Providencia & 1547 & 40,60 & San Andrés & 1192 & 62,5 & ND & & \\
\hline
\end{tabular}

Fuente: elaboración de los autores con base en la referencia 2. ND: Datos no disponibles. 
células de línea transfectada a partir de la línea $L$ de ratón con el clon ADN (20B) del receptor humano para poliovirus (L20B), las cuales son especialmente permisivas para el crecimiento de los poliovirus y excluyentes de la mayoría de los otros enterovirus. Estas células proceden del National Institute for Biological Standards and Control de Inglaterra y fueron donadas por el laboratorio de cultivo celular del Instituto de Medicina Tropical "Pedro Kourí", La Habana, Cuba. Para el aislamiento viral se siguieron los procedimientos generales recomendados por la OMS (10).

Los cultivos celulares que mostraron efecto citopático (ECP) se identificaron mediante pruebas de neutralización del efecto viral y reacción en cadena de la polimerasa convencional posterior a la transcripción reversa (TR-RCP). La técnica de neutralización del ECP se realizó con sueros equinos hiperinmunes a enterovirus $(\mathrm{A}-\mathrm{H})$, según el esquema de Lim Benyesh-Melnick (LBM), que permite identificar 42 serotipos diferentes (11). La TR-RCP se realizó a partir de ARN extraído de $250 \mu L$ del sobrenadante de cultivo celular mediante el método del TRIZOL (Life Technologies, Gibco BRL; Grand Island, N.Y., USA), siguiendo las indicaciones del fabricante. La amplificación del ARN se realizó siguiendo los procedimientos generales recomendados por la OMS (10). Se emplearon cuatro juegos de cebadores: i) genéricos (Pan EV) que hibridan con sitios altamente conservados dentro de la región 5' no codificante del genoma de los enterovirus, ii) específicos del grupo de los poliovirus
(Pan PV), iii) específicos de serotipo (PV) y iv) específicos de las cepas vacunales (Sabin 1, 2 y 3) (10).

Los resultados de la diferenciación intratípica de los aislamientos de poliovirus se confirmaron mediante reacción en cadena de la polimerasa en tiempo real ( $r$ TR-RCP) en el Laboratorio de Polio/ EV del Instituto Nacional de Salud, Bogotá, Colombia, siguiendo las instrucciones del protocolo de los Centros para el Control y la Prevención de Enfermedades (CDC, por sus siglas en inglés), Atlanta, Estados Unidos. Se utilizaron sondas tipo TAQ MAN e iniciadores que flanquean fragmentos de las regiones VP1 y 3D del genoma de los poliovirus. Se empleó un termociclador Applied Biosystems $7500^{\circledR}$, con la programación correspondiente al protocolo utilizado (12). Se realizó la secuencia completa de la región VP1 del genoma viral de los poliovirus aislados para determinar el porcentaje de diferencia nucleotídica con relación a la cepa vacunal Sabin. La secuenciación y el análisis filogenético se realizaron en el laboratorio global de polio de los CDC, empleando metodologías ya publicadas (13). De acuerdo al porcentaje de diferencia nucleotídica de las cepas en la región de la proteína capsidica VP1 con respecto a la cepa vacunal de poliovirus, se consideraron cepas vacunales aquellas que difieren menos de 1\%, VDPV las que difieren de $1 \%$ a $15 \%$ y cepas de poliovirus salvajes aquellas que difieren más de 15\% (14). En 11 (21\%) de las 52 muestras de aguas concentradas e inoculadas en cultivos celulares se obtuvo ECP característico de enterovirus (células redondeadas, refringentes, desprendimiento celular) en células RD. Se obtuvo ECP en L20b en dos de las 11 muestras positivas en RD.

Los aislamientos obtenidos en L20B se identificaron como poliovirus 2 por neutralización del ECP. La diferenciación intratípica mediante TR-RCP con cebadores específicos de cepa Sabin reveló el carácter vacunal de las cepas y fueron confirmadas como Sabin 2 mediante rTR-RCP. La secuenciación completa de la región del genoma que codifica para la proteína capsídica VP1 demostró en ambas cepas aisladas más de $99 \%$ de similitud de secuencia con las cepas OPV Sabin 2. Ambos aislamientos correspondieron al departamento del Guaviare (cuadro 2).

El resto de los aislamientos obtenidos en RD fueron confirmados como enterovirus mediante la amplificación del genoma con cebadores genéricos de enterovirus. Los enterovirus aislados correspondieron a los municipios de Caldas, Guainía, Guavaire, Nariño y Vichada. Se identificaron tres aislamientos como coxsackievirus B1, echovirus $30 \mathrm{y}$ echovirus 11. Los restantes seis enterovirus aislados no se neutralizaron con ninguno de los sueros equinos hiperinmunes a enterovirus $(\mathrm{A}-\mathrm{H})$ contenidos en la mezcla de sueros de Lim BenyeshMelnick, por lo que fueron tipificados como enterovirus no polio (cuadro 2).

Si bien las coberturas de vacunación inferiores a $80 \%$ en las poblaciones residentes de los departamentos estudiados podrían mantener la circulación del poliovirus, en las aguas residuales no se detectó ningún VDPV ni poliovirus

CUADRO 2. Resultados del aislamiento e identificación en los municipios donde se detectó enterovirus, Colombia, 2010

\begin{tabular}{|c|c|c|c|c|c|c|}
\hline Departamento & Municipio & ECP RD & ECP L20B & TR-RCP & NT & SECVP1 \\
\hline \multirow[t]{2}{*}{ Caldas } & Neira & Pos & $\mathrm{Neg}$ & Enterovirus & CVB1 & ND \\
\hline & Marulanda & Pos & $\mathrm{Neg}$ & Enterovirus & E11 & ND \\
\hline \multirow[t]{2}{*}{ Nariño } & Pasto & Pos & $\mathrm{Neg}$ & Enterovirus & EVNP & ND \\
\hline & Buesaco & Pos & $\mathrm{Neg}$ & Enterovirus & EVNP & ND \\
\hline Vichada & Puerto Carreño & Pos & Neg & Enterovirus & E30 & ND \\
\hline Guainía & Puerto Inírida & Pos & Neg & Enterovirus & EVNP & ND \\
\hline \multirow[t]{5}{*}{ Guaviare } & San José del Guaviare 1 & Pos & $\mathrm{Neg}$ & Enterovirus & EVNP & ND \\
\hline & San José del Guaviare 2 & Pos & $\mathrm{Neg}$ & Enterovirus & EVNP & ND \\
\hline & San José del Guaviare 3 & Pos & Neg & Enterovirus & EVNP & ND \\
\hline & El Retorno 1 & Pos & Pos & Sabin 2 & Polio 2 & Sabin 2 \\
\hline & El Retorno 2 & Pos & Pos & Sabin 2 & Polio 2 & Sabin 2 \\
\hline
\end{tabular}

Fuente: elaboración de los autores.

ECP: Efecto citopático; RD: células de rabdomiosarcoma humano; L20B: células de línea transfectada a partir de la línea $L$ de ratón con el receptor humano para poliovirus; TR-RCP: reacción en cadena de la polimerasa convencional posterior a la transcripción reversa; NT: Neutralización del efecto citopático; SECVP1: Secuencia VP1; Pos: Positivo; Neg: Negativo; CVB1: Coxsackievirus B1; E11: Echovirus

11; E30: Echovirus 30; EVNP: Enterovirus no polio; ND: Datos no disponibles. 
salvaje. En 17,3\% de las muestras se detectaron enterovirus no polio, así como la circulación de la cepa vacunal Sabin 2, lo cual sustenta la capacidad del método empleado para la detección viral en el ambiente.

Los porcentajes de enterovirus no polio detectados y los serotipos circulantes están en correspondencia con los hallados en estudios previos realizados en Colombia (15). Sin embargo, los poliovirus vacunales solo se detectaron en $3,8 \%$ de las muestras estudiadas. El bajo porcentaje de detección de poliovirus vacunales en un lugar donde se está utilizando la OPV, pero con coberturas de vacunación inferiores a $80 \%$, sugiere que los niveles de detección de poliovirus en el ambiente están en correspondencia con las coberturas de vacunación administradas. Esta hipótesis se puede sustentar en los resultados previos obtenidos en el departamento del Quindío, donde la cobertura de inmunización antipolio es superior a $80 \%$ y se detectó presencia de poliovirus vacunales en $55 \%$ de las muestras estudiadas empleando la misma metodología de concentración de aguas residuales y detección viral (datos no publicados).

Por otra parte, estudios realizados en Cuba con métodos similares a los utilizados en el presente trabajo demostraron la presencia de poliovirus vacunales en $100 \%$ de las aguas residuales colectadas a las 3 y 4 semanas después de concluidas las campañas de vacunación masiva con OPV (16). Como resultado de estas campañas masivas, los niveles de circulación de poliovirus al momento en que se realizó el estudio en Cuba fueron mayores que los que puedan existir en Colombia, donde no se realizaron campañas de vacunación masiva durante el tiempo de este estudio, lo que avala que los porcentajes de detección de poliovirus vacunal en el ambiente están en correspondencia con la cantidad de vacuna administrada.

Un factor que se debe tener en cuenta es el tiempo de transporte de algunas de las muestras de sitios alejados, tomando hasta 48 horas desde su recolección hasta el lugar de procesamiento, circunstancia que pudo haber influido en los resultados. Este factor, junto con las bajas coberturas de vacunación, podrían justificar los bajos porcentajes de poliovirus vacunales hallados. A pesar de estas posibles limitaciones, los resultados sugieren la ausencia de circulación de poliovirus en departamentos colombianos con coberturas vacunales inferiores a $80 \%$.

No obstante, el nivel de cobertura de vacunación que se requiere para prevenir la circulación de poliovirus salvaje y VDPV no se conoce con exactitud. De allí que sea necesario fortalecer las coberturas vacunales en los municipios en riesgo, así como realizar investigaciones sobre respuesta inmune a poliovirus en la población infan-

\section{REFERENCIAS}

1. Bonet MC, Dutta A. World wide experience with inactivated poliovirus vaccine. Vaccine. 2008;26(39):4978-83.

2. Ministerio de Salud y Protección Social, Colombia. Disponible en: http://www.minproteccion social.gov.co/ Acceso el 20 de junio de 2012.

3. Colombia, Ministerio de la Protección Social; Organización Panamericana de la Salud. Cruzada interminable por la niñez colombiana: Historia del Programa Ampliado de Inmunizaciones-PAI-En Colombia. 1979-2009. Colombia: MPS/OPS; 2010.

4. González MM, Sarmiento L, Castaño JC, Giraldo AM, Salazar AR, Muñoz NJ. Detección de poliovirus en aguas residuales de Armenia, Colombia. Rev Salud Publica (Bogota). 2006;813-23.

5. Centers for Disease Control and Prevention. Tracking progress toward global polio eradication-worldwide, 2009-2010. MMWR Morb Mortal Wkly Rep. 2011;60:441-5.

6. Centers for Disease Control and Prevention. Outbreaks following wild poliovirus importations-Europe, Africa and Asia, January 2009-September 2010. MMWR Morb Mortal Wkly Rep. 2010;59:1393-9.
7. Kew OM, Sutter RW, de Gourville EM, Dowdle WR, Pallansch MA. Vaccine-derived polioviruses and the endgame strategy for global polio eradication. Annu Rev Microbiol. 2005;59:587-635.

8. World Health Organization. Guidelines for environmental surveillance of poliovirus circulation. Vaccine \& Biologicals. Geneva: WHO; 2003.

9. Sobsey M. Methods for recovering viruses from shellfish, seawater and sediments. In: Berg G, ed. Methods for recovering viruses in the environments. Boca Ratón, FL: CRC Press; 1987. Pp.77-108.

10. World Health Organization. Polio laboratory manual. Geneva: WHO; 2004.

11. Melnick JL, Wimber IL. Liophilized combination of the Enterovirus equine antisera. New LBM pools prepared from reserves of antisera stored frozen for two decades. Bull World Health Organ. 1984;63:543-50.

12. Kilpatrick DR, Yang CF, Ching K, Vincent A, Iber J, Campagnoli R, et al. Rapid group-, serotype-, and vaccine strain-specific identification of poliovirus isolates by real-time reverse transcription-PCR using degenerate primers til colombiana para determinar el nivel de protección en estos departamentos y corroborar la correspondencia de tal protección con las coberturas administrativas. Estos estudios permitirán evaluar la vulnerabilidad de estas poblaciones a la infección por poliovirus, una medida de gran utilidad como paso previo para iniciar el cambio de la vacuna oral a la inactivada. Finalmente, también harán falta estudios que incluyan zonas de Colombia con coberturas de inmunización tanto altas como bajas, dirigidos a estimar la sensibilidad de la vigilancia ambiental en diferentes entornos y su posible introducción en otros países de América Latina y el Caribe.

Agradecimientos. Los autores quieren expresar su gratitud a todas las personas que los apoyaron durante el proceso de toma de muestras en los nueve departamentos visitados: Boyacá, Caldas, Cauca, Guainía, Guaviare, Nariño, Risaralda, San Andrés y Providencia y Vichada. Agradecen asimismo a Dioselina Pelaez, del Instituto Nacional de Salud Colombia, por su apoyo en la diferenciación intratípica de los aislados.

Financiación. El presente trabajo fue financiado por Colciencias y por la $\mathrm{Vi}$ cerrectoría de Investigaciones de la Universidad del Quindío, Colombia. and probes containing deoxyinosine residues. J Clin Microbiol. 2009;47:1939-41.

13. Liu M, Zheng DP, Zhang LB, Oberste MS, Pallansch MA, Kew OM. Molecular evolution of a type 1 wild-vaccine poliovirus recombinant during widespread circulation in China. J Virol. 2000;74:11153-61.

14. DeVries A, Harper J, Murray A, Lexau C, Bahta L, Christensen J, et al. Vaccine-Derived Poliomyelitis 12 Years after Infection in Minnesota. N Engl J Med. 2011;364:2316-23.

15. González M, Castaño J, Giraldo A, Quintero L, Sarmiento L. Prevalence of enterovirus in newborns and infants who consult a first-class Center, Armenia, Colombia 2009. Biomedicas. 2011;31(4).

16. Mas P, Gary H, Sarmiento L, Caceres V, Barrios J, Palomera R, et al. Poliovirus detection in wastewater and stool following an immunization campaign in Havana, Cuba. Int J Epidemiol. 2003;32:772-7.

Manuscrito recibido el 21 de junio de 2011. Aceptado para publicación, tras revisión, el 23 de mayo de 2012. 
ABSTRACT This study aims to explore a possible silent circulation of wild and vaccine-derived polioviruses in departments of Colombia with polio vaccination coverage of below $80 \%$. The study collected 52 samples of wastewater concentrated as a result of

\section{Absence of poliovirus circulation in Colombian departments with vaccination coverage below $80 \%$} precipitation with polyethylene glycol and sodium chloride. The viral detection was carried out through isolation and the identification through neutralization of the cytopathic effect, as well as through a conventional polymerase chain reaction following reverse transcription. The isolated polioviruses were characterized by the VP1 gene sequence. In two of the 52 samples, there was a presence of the Sabin type 2 poliovirus with more than $99 \%$ sequence similarity with the Sabin type 2 strain polio. Circulation of the nonpolio enterovirus was detected in $17.3 \%$ of the samples. The serotypes identified corresponded to coxsackievirus B1, echovirus 30, and echovirus 11. No evidence of the spread of either vaccine-derived poliovirus or wild poliovirus was detected in the departments of Colombia with polio coverage lower than $80 \%$.

Key words Poliomyelitis; poliovirus vaccine, oral; poliovirus vaccines; immunization coverage; Colombia. 